Research Article

\title{
Treatment Efficacy of Chuang Ling Ye, a Traditional Chinese Herbal Medicine Compound, on Idiopathic Granulomatous Mastitis: A Randomized Controlled Trial
}

\author{
Jing-xian Xue $\mathbb{D}$, Bei Ye, Shun Liu $(\mathbb{D}$, Si-han Cao, Wei-he Bian, and Chang Yao $\mathbb{D}$ \\ Affiliated Hospital of Nanjing University of Chinese Medicine, Nanjing 210029, China \\ Correspondence should be addressed to Chang Yao; yaochang196705@163.com
}

Received 24 February 2020; Accepted 26 May 2020; Published 29 June 2020

Guest Editor: Xiangguo Shi

Copyright (c) 2020 Jing-xian Xue et al. This is an open access article distributed under the Creative Commons Attribution License, which permits unrestricted use, distribution, and reproduction in any medium, provided the original work is properly cited.

\begin{abstract}
Objective. To explore whether Chuang Ling Ye (CLY), a traditional Chinese herbal medicine compound, could improve the treatment of idiopathic granulomatous mastitis (IGM) via decreasing inflammatory response. Methods. Herein, 40 patients with IGM who had wounds requiring dressing change were enrolled and randomly divided into two groups: the CLY group and the control group. The size of the neoplasm and pain score of patients were followed-up for 4 weeks. Local tissues were taken during dressing change and examined by commercial enzyme-linked immunosorbent assay (ELISA) kits. The levels of inflammatory markers, including interleukin-1 $\beta$ (IL-1 $\beta$ ), IL-2, IL-6, interferon gamma (IFN- $\gamma$ ), and tumor necrosis factor- $\alpha$ (TNF- $\alpha$ ) were measured. Results. After treatment, the size of the neoplasm in the CLY group was significantly smaller than that in the control group $(14.28 \mathrm{~cm} \pm 8.96 \mathrm{~cm}$ vs. $21.14 \mathrm{~cm} \pm 0.12 \mathrm{~cm}, P=0.038)$, and the pain scores were markedly reduced $(P=0.004)$. Besides, CLY downregulated the expression levels of IL- $1 \beta$, IFN- $\gamma$, and TNF- $\alpha$. Conclusion. External use of CLY could reduce the neoplasm of IGM by inhibiting local inflammation. This trial is registered with ChiCTR1800017744.
\end{abstract}

\section{Introduction}

Idiopathic granulomatous mastitis (IGM) is a rare benign inflammatory breast disease with an estimated incidence of 2.4 per 100,000 women and $0.37 \%$ in the United States, while its incidence is higher among Asian populations [1]. The leading symptom of IGM is a painful neoplasm. Other symptoms are hyperemia, areolar, fistula, and ulceration [2]. IGM is diagnosed by histopathology only. The disease is characterized by formation of granuloma in combination with a localized infiltrate of multinucleated giant cells, lymphocytes, and plasma cells [3]. The etiopathogenesis of IGM reported in the literature included breast dysplasia, nipple deformity, smoking, and taking psychotropic drugs, leading to abnormal secretion of lipid substances in mammary duct endothelium, blockage of ducts caused by accumulation of secretions, and chronic inflammation [4], which suggested that inhibiting inflammation may be effective in the treatment of IGM.
In modern medicine, management of IGM cases remains controversial with proponents of initial surgical or medical therapies and each one has its associated complications, which may be worse than the original symptoms of IGM [5]. The main treatment option for IGM is surgery. In acute stage, antibiotics and surgical incision can be used, and the surgery can be performed after the neoplasm shrinks. If the neoplasm is small, local resection of the neoplasm can be carried out; if the neoplasm is large, a simple mastectomy can be conducted as well [6]. Versluijs-Ossewaarde et al. [7] found that $79 \%$ of patients were treated without removing the diseased catheter, while $28 \%$ were treated after removing the diseased catheter. Therefore, the main target of surgical treatment is to completely and thoroughly remove the lesions in the large lacteal duct avoiding recurrence. Lei et al. [8] demonstrated that surgical management have a high complete remission/resolution (CR) rate with a relatively low recurrence rate, with or without steroids. Thus, it is highly appropriate for patients requiring rapid remission. 
However, for patients with concerns about surgical scarring, oral steroids could be an acceptable option.

Traditional Chinese medicine (TCM) has significantly attracted scholars' attention for treating breast diseases [9]. For instance, Chuang Ling Ye (CLY), a traditional Chinese herbal medicine compound, is composed of rhubarb, safflower, Abelmoschus manihot, and Terminalia chebula. CLY has been used in clinical practice for promoting wound healing and reducing wound secretion since the 1990s. A previous study found that CLY has an appropriate antiinflammatory effect, as well as an ability to promote angiogenesis [10-12]. We, in the present research, attempted to explore whether CLY could improve the treatment of IGM via reducing inflammatory response.

\section{Patients and Methods}

2.1. Design. This was a randomized controlled trial (RCT) and was conducted in accordance with the CONSORT-SPI 2018 checklist. Eligible patients were randomized 1:1 to either the CLY group or control group.

\subsection{Study Subjects}

\subsubsection{Inclusion Criteria}

(i) Patients who were pathologically confirmed with IGM

(ii) Patients who aged 18-60 years old

(iii) No hormone or immunosuppressive therapy was used within one month before admission

\subsubsection{Exclusion Criteria}

(i) Pregnant or lactating women

(ii) Patients with allergic constitution or allergy to CLY

(iii) Patients who would like to withdraw consent, or if they failed to adhere to the research protocol, the individual's participation was suspended, or withdrawal was recorded

\subsection{Intervention}

2.3.1. CLY Group. After incision and drainage or self-ulceration of abscess, treatment was conducted for 4 weeks, including wound dressing with $30 \mathrm{ml}$ CLY that flushed and drained with CLY gauze strip twice a week.

2.3.2. Control Group. After incision and drainage or selfulceration of abscess, treatment was carried out for 4 weeks, involving wound dressing with $30 \mathrm{ml}$ saline that flushed and drained with saline gauze strip twice a week.

2.3.3. Preparation of CLY. CLY was herein composed of $200 \mathrm{~g}$ of rhubarb, $100 \mathrm{~g}$ of safflower, $50 \mathrm{~g}$ of Abelmoschus manihot, and $100 \mathrm{~g}$ of Terminalia chebula (Table 1). Among the abovementioned four flavors, Abelmoschus manihot was heated and refluxed twice with $75 \%$ ethanol for $1.5 \mathrm{~h}$ each time. For the other three flavors, water was added and decoction was done twice. After that, the filtrate was combined, it was concentrated to the extract with a relative density of $1.15\left(80^{\circ} \mathrm{C}\right)$, and ethanol was added to make the alcohol content $70 \%$. Then, it was allowed to settle, the supernatant was taken to recover ethanol, and it was concentrated to about $900 \mathrm{~mL}$. The Abelmoschus manihot was added; while it is hot, it was mixed well and settled for $24 \mathrm{~h}$, and water was added to $1000 \mathrm{~mL}$ [12].

2.3.4. Quality Control. Analysis of components of CLY was carried out by ultraperformance liquid chromatography-tandem mass spectrometry (UPLC-MS/MS). Accurately, $1 \mathrm{~mL}$ of CLY was measured, it was concentrated to dryness under vacuum, and it was dissolved into $10 \mathrm{~mL}$ to obtain the test solution. After shaking the test solution, it was centrifuged at $16,000 \mathrm{rpm}$ for $10 \mathrm{~min}$, it was filtered with a $0.22 \mu \mathrm{M}$ pore size filter, $190 \mu \mathrm{L}$ of continuous filtrate was taken, and $10 \mu \mathrm{L}$ of caffeic acid was added. The mobile phase consisted of acetonitrile and $0.2 \%$ ammonium acetate. The gradient program was produced by increasing the rate of acetonitrile from $25 \%$ to $80 \%$ and decreasing the rate of ammonium acetate from $75 \%$ to $20 \%$ for $13 \mathrm{~min}$. Mass spectrometry analyses were performed on a UHPLC system (Acquity H-Class, Waters Corp., Milford, MA, USA) with a binary solvent manager, a column manager, and a sample manager. The sample was separated on a Waters Acquity HSS T3 column $(100 \mathrm{~mm} \times 2.1 \mathrm{~mm}, 1.8 \mu \mathrm{m}$, Waters Corp.), and the column temperature was set at $40^{\circ} \mathrm{C}$. The mobile phase consisted of acetonitrile (A) and water containing $0.05 \%$ formic acid (B) with a flow rate of $0.25 \mathrm{~mL} / \mathrm{min}$. For UHPLCMS/MS analysis, a Waters QqQ-MS (Xevo TQ-D, Waters Corp.) was connected to the Waters UHPLC instrument via an electrospray ionization (ESI) source. Analytes were quantified by multiple reaction monitoring (MRM) in negative ionization mode with argon as the collision gas.

\subsection{Outcome Measures}

2.4.1. Primary Outcome Measures. Neoplasm size was measured by the area of the neoplasm. The area of the neoplasm was photographed at the time of treatment. Finally, the Image J 16.0 software was used to measure the area of the neoplasm.

2.4.2. Pain Score. The Visual Analog Scale pain scoringsystem was employed, in which 10-point indicated the most painful condition, while 0 -point denoted no painful condition.

2.4.3. Inflammatory Factors. At days 1, 15, and 29, the levels of inflammatory factors, such as interleukin-1 $\beta$ (IL-1 $\beta$ ), IL-2, IL-6, interferon gamma (IFN- $\gamma$ ), and tumor necrosis factor$\alpha$ (TNF- $\alpha$ ) were measured using commercial enzyme-linked immunosorbent assay (ELISA) kits (Hangzhou Multisciences Biotech Co, Ltd., Hangzhou, China). 
TABLE 1: CLY composition.

\begin{tabular}{|c|c|c|c|}
\hline $\begin{array}{l}\text { Name (botanical, } \\
\text { common Pinyin names), } \\
\text { traditional daily dose } \\
\text { (grams) }\end{array}$ & Active compounds & Clinical and pharmacological effects & Adverse effects/toxicity \\
\hline $\begin{array}{l}\text { Abelmoschus manihot } \\
\text { (L.) Medik, Huang } \\
\text { Shukuihua, 10-30g }\end{array}$ & $\begin{array}{l}\text { The known chemical } \\
\text { component of Abelmoschus } \\
\text { manihot is mainly } \\
\text { hyperoside [13] }\end{array}$ & $\begin{array}{l}\text { Abelmoschus manihot treats some } \\
\text { kidney diseases. It protects renal tubular } \\
\text { cells via inhibition of ROS-ERK1/2- } \\
\text { NLRP3 pathway [13-15] }\end{array}$ & No reported adverse events \\
\hline $\begin{array}{l}\text { Rheum palmatum L., } \\
\text { rhubarb root and } \\
\text { rhizome, Da Huang, } \\
3-15 \mathrm{~g}\end{array}$ & $\begin{array}{l}\text { The rhubarb root contains } \\
\text { aloe-emodin, rhein, emodin, } \\
\text { chrysophanol et al. [16] }\end{array}$ & $\begin{array}{c}\text { It has been used for a variety of } \\
\text { conditions, such as constipation, } \\
\text { chronic renal failure, and upper } \\
\text { gastrointestinal bleeding. It has } \\
\text { antimicrobial and antioxidative effects } \\
{[17,18]}\end{array}$ & $\begin{array}{l}\text { The toxicity includes hepatotoxicity } \\
\text { and nephrotoxicity. No adverse } \\
\text { reactions were observed in mice with a } \\
\text { dose of } 400 \mathrm{mg} / \mathrm{kg} \text { [19] }\end{array}$ \\
\hline $\begin{array}{l}\text { Carthamus tinctorius L., } \\
\text { safflower, Hong Hua, } \\
3-10 \mathrm{~g}\end{array}$ & $\begin{array}{l}\text { The flowers of safflower } \\
\text { contain kaempferol and } \\
\text { hydroxysafflor yellow [20] }\end{array}$ & $\begin{array}{l}\text { It reduces immune inflammatory } \\
\text { response [14]. Carthamus tinctorius } \\
\text { (safflower) oil is used as a dietary } \\
\text { supplement for weight loss and } \\
\text { antioxidant [21] }\end{array}$ & $\begin{array}{l}3 \text { patients had a diagnosis of acute liver } \\
\text { failure, while consumed safflower as a } \\
\text { dietary supplement for weight loss [21] }\end{array}$ \\
\hline $\begin{array}{l}\text { Terminalia chebula Retz., } \\
\mathrm{Ke} \mathrm{Zi}, 3-10 \mathrm{~g}\end{array}$ & $\begin{array}{l}\text { Contains chebulin and } \\
\text { hyperoside }[16,22]\end{array}$ & $\begin{array}{l}\text { It is used in asthma, ulcers, gout, heart, } \\
\text { and bladder diseases [23] }\end{array}$ & No reported adverse events \\
\hline
\end{tabular}

2.5. Sample Size. We collected baseline data of 10 IGM patients who were treated in our hospital with CLY and were recovered after 3 mouths, in addition to data extracted from previous studies (Chirapapha et al., 2018, [2]). To account for a potential drop-out rate of up to $20 \%$ at 28 days, we planned to randomize 45 patients.

2.6. Randomization. Patients were randomly assigned to study groups by nonstratified randomization. The random sequence was generated using the random number function of Microsoft ${ }^{\circledR}$ Excel software. The randomization list was kept on a password-secured computer. All patients, study site personnel, raters, and contract research organization staff were blinded to group assignment.

2.7. Research Ethics and Informed Consent. The research protocol was approved by the Institutional Review Board of the Affiliated Hospital of Nanjing University of Traditional Chinese Medicine (Nanjing, China). From September 2018 to October 2019, patients who were admitted from the Breast Disease Department of our hospital were screened and enrolled in the current study, and all the eligible patients signed the written informed consent form prior to commencing the study. The research protocol was registered at the Chinese Clinical Trial Registry (reg. no. ChiCTR1800017744).

2.8. Statistical Analysis. Continuous data were compared using Student's t-test. Groups were compared using the t-test or Student's t-test, as appropriate based on the data distribution. Categorical variables were expressed as percentages. Groups were compared using the chi-square or Fisher's exact test, as appropriate based on the expected counts. All statistical analyses were performed using SPSS 17.0 software (IBM, Armonk, NY, USA).

\section{Results}

3.1. Analysis of the Main Medicinal Components of CLY. The main components of CLY were analyzed by UPLC-MS/ MS. CLY contained emodin $(0.09178 \mathrm{mg} / \mathrm{ml})$, rhein $(0.25078 \mathrm{mg} / \mathrm{ml})$, physcion $(0.15954 \mathrm{mg} / \mathrm{ml})$, aloe-emodin $(0.09472 \mathrm{mg} / \mathrm{ml})$, hydroxysafflor yellow A $(0.61545 \mathrm{mg} / \mathrm{ml})$, kaempferide $(0.001943 \mathrm{mg} / \mathrm{ml})$, and hyperoside $(0.92247 \mathrm{mg} / \mathrm{ml})$ (Figures 1 and 2$)$.

3.2. Patients'Baseline Characteristics. From September 2018 to October 2019, 45 patients were screened for eligibility and 5 of whom were ineligible (Figure 3). Of the remaining 40 patients, 20 were assigned to the CLY group and 20 to the control group. In the CLY group, 4 patients received wound healing at day 29, while 2 patients in the control group received wound healing at day 29 . The patients' age ranged from 26 to 50 years old, and they all were female. There was no significant difference in the patients' age, weight, and breastfeeding month between the two groups. During the treatment period (within 4 weeks), no oral hormones or antibiotics were given to the two groups (Table 2).

3.3. Size of Neoplasm. There were neoplasms in the two groups before treatment. At day 1, the size of the neoplasm in the control group was $34.2-48.3 \mathrm{~cm}^{2}$, and that was $34.0-47.4 \mathrm{~cm}^{2}$ in the CLY group. There was no significant difference in the size of the neoplasm between the two groups $(P=0.865)$. At day 15 , both groups had a smaller neoplasm than before. At day 29, the size of the neoplasm in the CLY group was remarkably smaller than that in the control group $(P=0.001<0.05)$. This suggested that IGM was a self-healing disease. In the presence of wound (natural rupture or incision and drainage), regular dressing change and timely drainage could reduce the size of the neoplasm. 


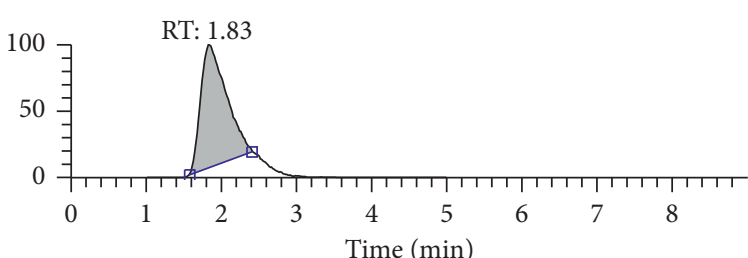

(a)

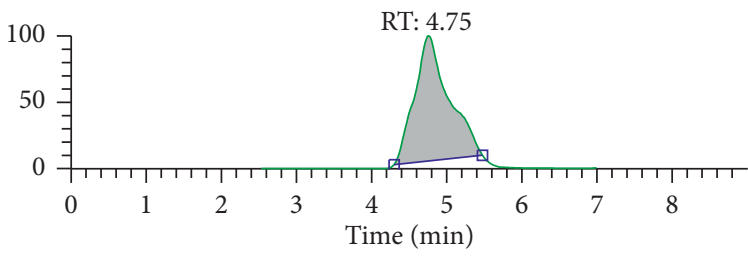

(c)

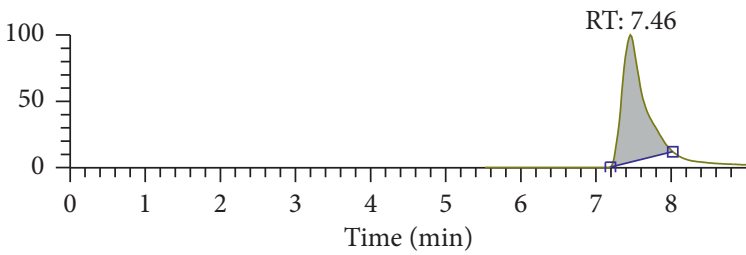

(e)

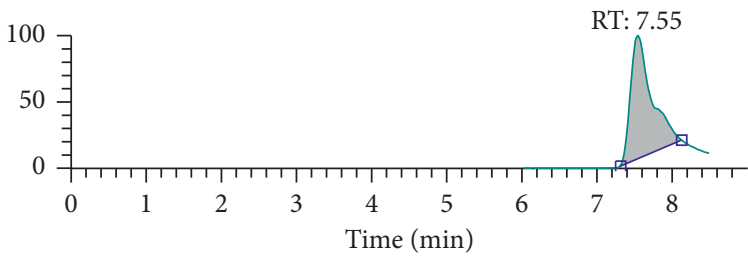

(g)

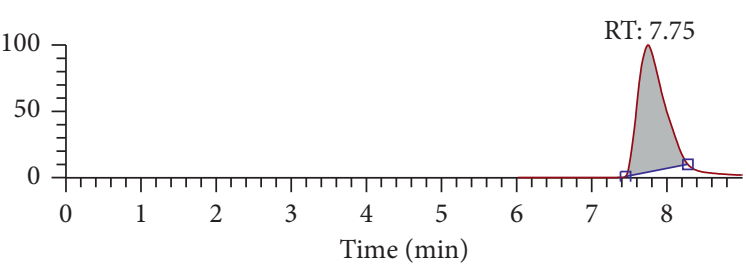

(b)

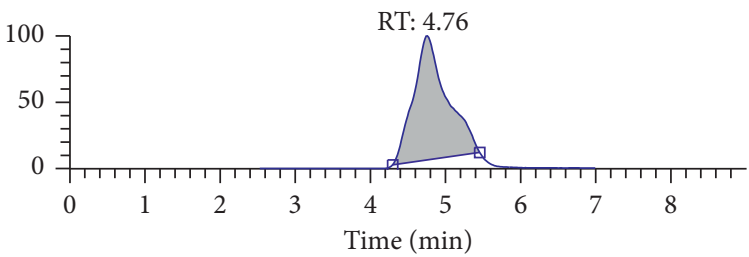

(d)

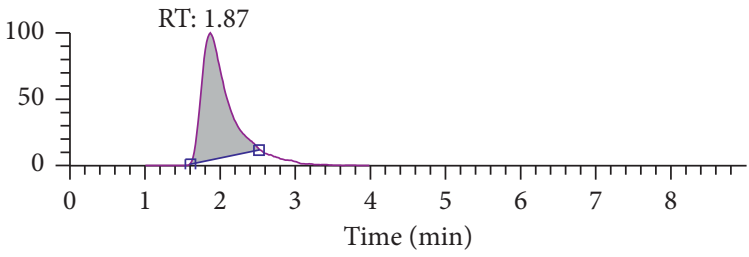

(f)

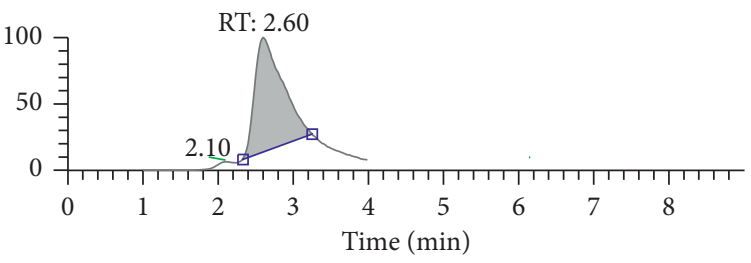

(h)

Figure 1: Analysis of the main components of CLY. (a) Caffeic acid, (b) emodin, (c) rhein, (d) physcion, (e) aloe-emodin, (f) hydroxysafflor yellow A, (g) kaempferide, and (h) hyperoside.

The use of CLY during dressing change could markedly decrease the size of the neoplasm $\left(14.28+8.96 \mathrm{~cm}^{2}\right.$ vs. $21.14+10.12 \mathrm{~cm}^{2}, P=0.038$ ) (Table 3 ).

3.4. Pain Score. In the current study, we found that the patients' mean pain score was 6-point before treatment. Pain affected patients' daily life. There was no significant difference in pain score between the two groups at day 1 $(P=0.829)$. After treatment, the pain score significantly decreased, which was consistent with the trend of decreasing the size of the neoplasm. After treatment, the pain score in the CLY group was noticeably lower than that in the control group $(P=0.004<0.05)$, highlighting that the use of CLY had a relieving effect on the patients' pain (Table 4).

3.5. Inflammatory Factors. The findings of the present research revealed that there was no significant difference in the levels of IL- $1 \beta$, IFN- $\gamma$, and TNF- $\alpha$ between the two groups at day 1 , while a significant difference in the levels of these factors was noted between the control group and the CLY group after treatment. It was unveiled that CLY could reduce the neoplasm by inhibiting inflammation. IL-6 also plays a significant role in inflammatory response [11, 24]; however, there was no significant difference between the two groups after treatment in the present experiment $(P=0.978>0.05)$. The outcomes of the current research uncovered that there was no significant difference in IL-2 level between the two groups before treatment. After treatment, the $P$ value of the difference related to IL-2 level between the control group and the CLY group was 0.057 , and there were still some differences although that was greater than 0.05 (Table 5). Since CLY was composed of Abelmoschus manihot, it may play a role in immune response to IGM.

During treatment, there were patients who received wound healing in the two groups; thus, no samples were taken from these patients. There were 2 and 4 cases who received wound healing in the control group and the CLY group, respectively.

\section{Discussion}

IGM can mimic two very frequent breast disorders, breast carcinoma, and breast abscess. To date, a limited number of RCTs were reported, and the majority of publications were retrospective studies and case series $[25,26]$. A retrospective cohort study showed that there was no significant difference 


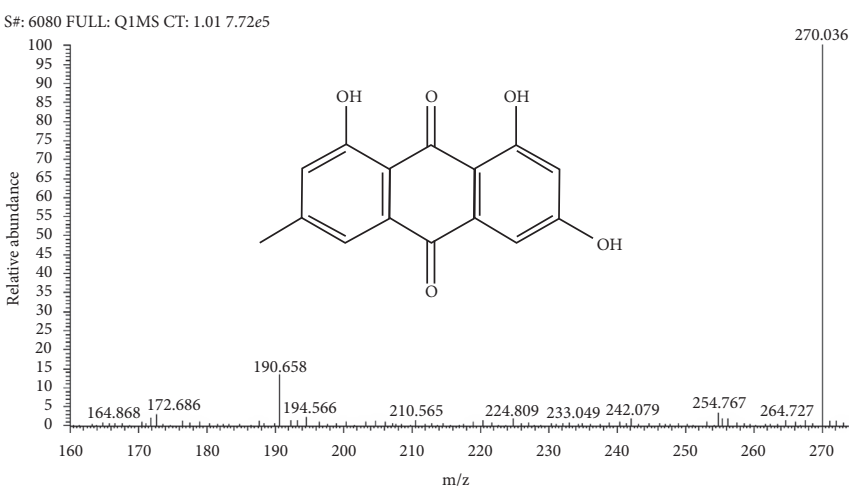

(a)

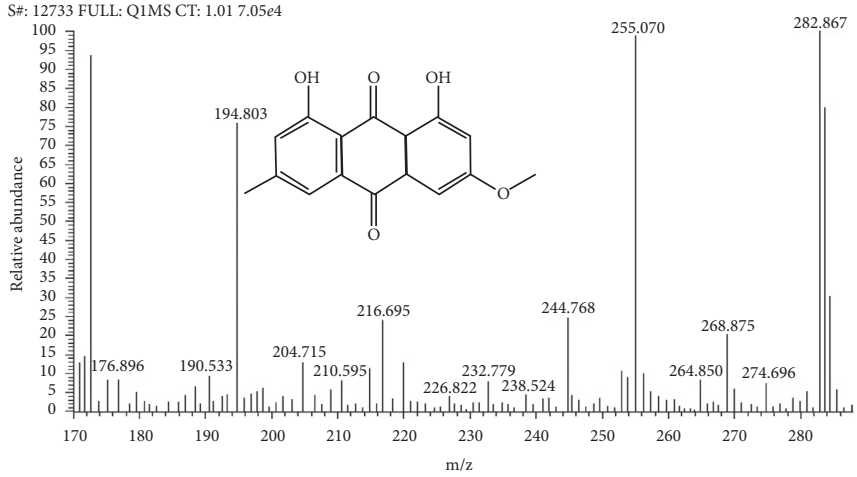

(c)

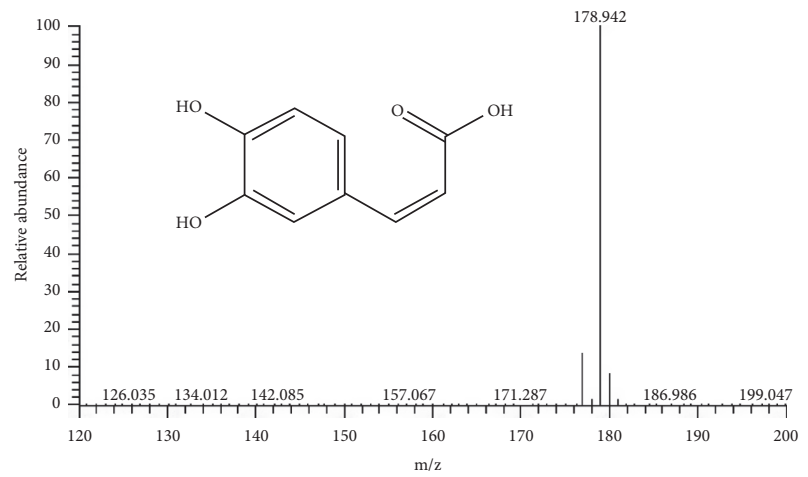

(e)

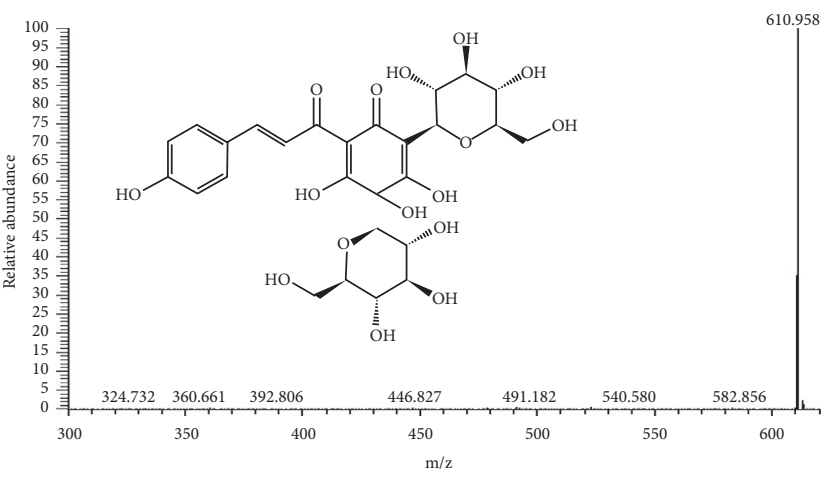

(g)

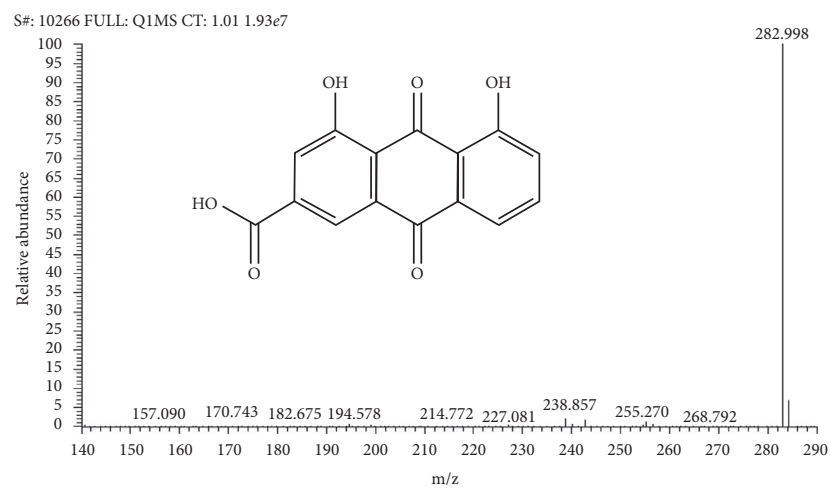

(b)

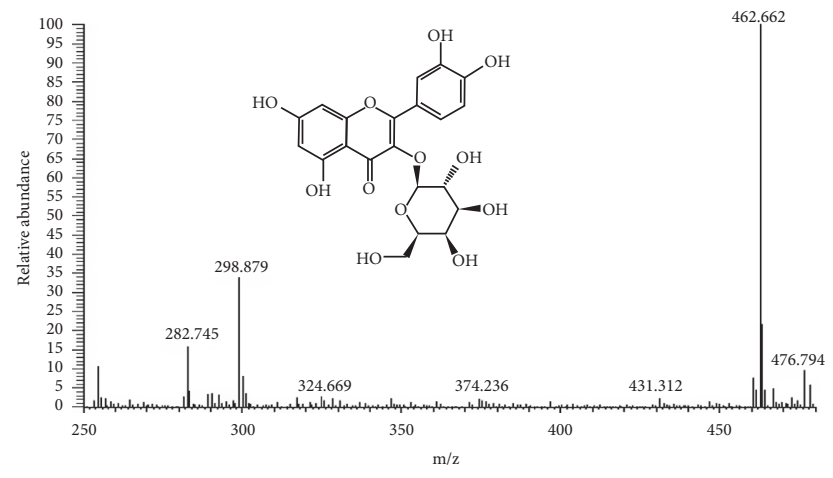

(d)

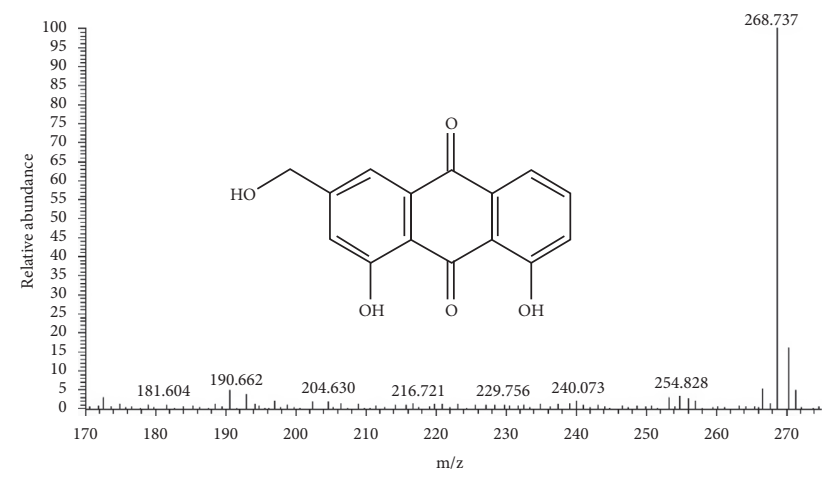

(f)

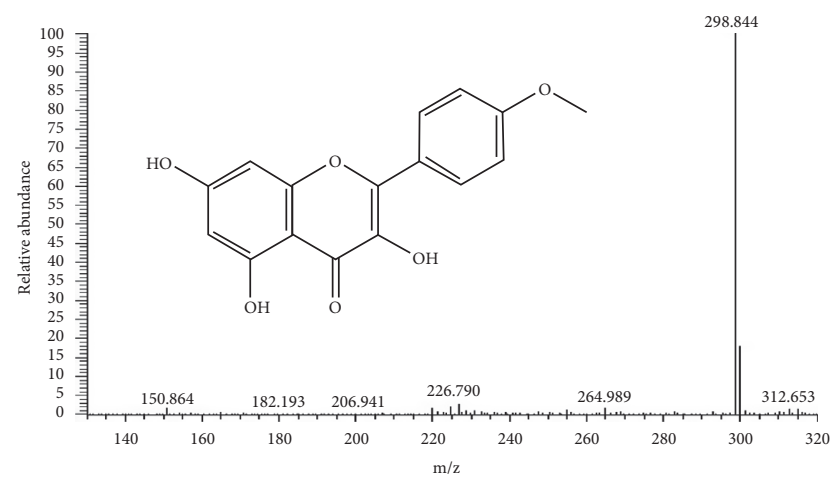

(h)

FIGURe 2: Chromatogram. (a) emodin, (b) rhein, (c) physcion, (d) hyperoside, (e) caffeic acid, (f) aloe-emodin, (g) hydroxysafflor yellow A, and (h) kaempferide. 


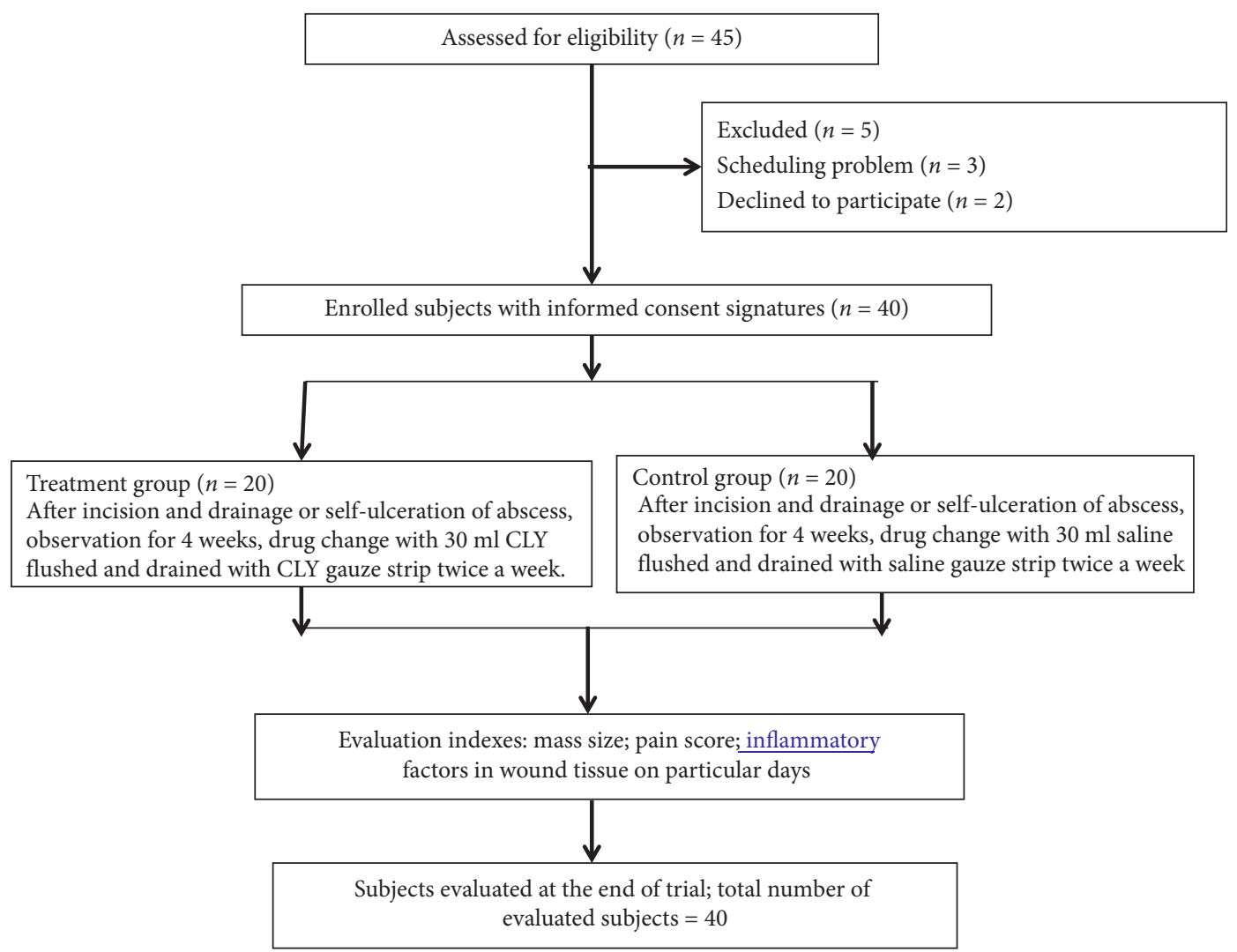

FIGURE 3: CONSORT followchart of the clinical study.

TABLE 2: Baseline characteristics of the participants.

\begin{tabular}{lccc}
\hline & Control group & CLY group & $P$ value \\
\hline Age (years) & $34.65 \pm 7.49$ & $34.35 \pm 4.42$ & 0.878 \\
Height $(\mathrm{cm})$ & $158.6 \pm 4.58$ & $159 \pm 3.6$ & 0.760 \\
Weight $(\mathrm{kg})$ & $57.7 \pm 6.81$ & $57.3 \pm 6.59$ & 0.851 \\
BMI $\left(\mathrm{kg} / \mathrm{m}^{2}\right)$ & $22.5 \pm 2.69$ & $21.78 \pm 2.36$ & 0.374 \\
Lactation (month) & $9.55 \pm 5.08$ & $8.95 \pm 4.55$ & 0.696 \\
\hline
\end{tabular}

TABLE 3: Comparing the size of the neoplasm (in $\mathrm{cm}^{2}$ ) between the two groups.

\begin{tabular}{lcccr}
\hline & Control group & CLY group & Estimated group difference (95\% confidence interval) & $P^{\text {a }}$ \\
\hline Day 1 & $41.09 \pm 4.00$ & $41.33 \pm 4.66$ & & 0.865 \\
Day 15 & $29.14 \pm 7.71$ & $27.46 \pm 9.25$ & $-1.92(-7.61,3.77)$ & 0.489 \\
Day 29 & $21.14 \pm 10.12$ & $14.28 \pm 8.96$ & $-7.1(-13.75,-0.45)$ & 0.038 \\
$P^{\text {b }}$ & 0.001 & 0.001 & & \\
\hline
\end{tabular}

${ }^{\mathrm{a}}$ Comparing the two groups. ${ }^{\mathrm{b}}$ Comparing at day 29 with that at day 1.

TABLE 4: Comparison of the pain score between the two groups.

\begin{tabular}{lcccc}
\hline & Control group & CLY group & Estimated group difference $(95 \%$ confidence interval) & $P$ value \\
\hline Day 1 & $5.45 \pm 1.00$ & $5.90 \pm 0.72$ & & 0.849 \\
Day 15 & $3.85 \pm 1.18$ & $3.50 \pm 1.05$ & $-0.5(-1.25,0.25)$ & 0.180 \\
Day 29 & $2.20 \pm 1.20$ & $1.15 \pm 0.88$ & $-1.10(-1.79,-0.41)$ & 0.004 \\
\hline
\end{tabular}

among treatment modalities in terms of time-to-healing and recurrence of disease (Chirapapha et al. 2018). The current RCT presented a new effective therapy for IGM. The results of the present research revealed that CLY could promote the shrinkage and healing of plasma cell mastitis and decrease the pain in the course of disease. Measurement of the levels of inflammatory factors unveiled that CLY could inhibit the levels of IL- $1 \beta$, IFN- $\gamma$, and TNF- $\alpha$. 
TABLE 5: Comparing the levels of inflammatory factors between the two groups.

\begin{tabular}{|c|c|c|c|c|c|}
\hline \multicolumn{2}{|c|}{$\begin{array}{c}\text { Inflammatory factor } \\
(\mathrm{pg} / \mathrm{ml})\end{array}$} & \multirow{2}{*}{$\begin{array}{l}\text { Control group } \\
248.34 \pm 53.56\end{array}$} & \multirow{2}{*}{$\begin{array}{l}\text { CLY group } \\
243.8 \pm 78.15\end{array}$} & \multirow[t]{2}{*}{ Estimated group difference (95\% confidence interval) } & \multirow{2}{*}{$\begin{array}{c}P \\
0.831\end{array}$} \\
\hline \multirow{3}{*}{ TNF- $\alpha$} & Day 1 & & & & \\
\hline & Day 15 & $264.19 \pm 95.67$ & $159.48 \pm 72.25$ & $-85.47(-149.65,-21.28)$ & 0.012 \\
\hline & Day 29 & $200.20 \pm 76.81$ & $110.95 \pm 56.91$ & $-81.59(-156.45,-6.73)$ & 0.035 \\
\hline \multirow{3}{*}{ IFN- $\gamma$} & Day 1 & $36.95 \pm 15.42$ & $50.41 \pm 37.06$ & & 0.145 \\
\hline & Day 15 & $36.81 \pm 15.64$ & $32.00 \pm 27.46$ & $-18.27(-41.20,4.65)$ & 0.112 \\
\hline & Day 29 & $38.8 \pm 23.53$ & $16.35 \pm 11.1$ & $-45.78(-68.58,-22.97)$ & 0.001 \\
\hline \multirow{3}{*}{ IL- $1 \beta$} & Day 1 & $767.11 \pm 235.88$ & $763.43 \pm 176.21$ & & 0.956 \\
\hline & Day 15 & $705.1 \pm 205.51$ & $370.56 \pm 177.94$ & $-0.50(-1.25,0.25)$ & 0.180 \\
\hline & Day 29 & $723.8 \pm 198.57$ & $248.47 \pm 119.62$ & $-1.10(-1.79,-0.41)$ & 0.004 \\
\hline \multirow{3}{*}{ IL-2 } & Day 1 & $224.91 \pm 97.41$ & $234.56 \pm 93.62$ & & 0.751 \\
\hline & Day 15 & $167.79 \pm 124.42$ & $111.96 \pm 87.89$ & $-65.48(-159.64,28.69)$ & 0.162 \\
\hline & Day 29 & $114.05 \pm 64.86$ & $48.47 \pm 27.53$ & $-89.27(-181.55,3.02)$ & 0.057 \\
\hline \multirow{3}{*}{ IL-6 } & Day 1 & $144.42 \pm 47.67$ & $132.38 \pm 60.68$ & & 0.489 \\
\hline & Day 15 & $88.06 \pm 39.60$ & $66.34 \pm 38.2$ & $-9.67(-50.02,30.67)$ & 0.622 \\
\hline & Day 29 & $50.64 \pm 20.12$ & $36.91 \pm 16.54$ & $-0.65(-49.53,48.23)$ & 0.978 \\
\hline
\end{tabular}

For the neoplasm treatment, there are several therapeutic strategies as follows, in which surgical treatment is the main method for a simple neoplasm (Chirapapha et al. 2018). However, several patients have a neoplasm with abscesses or fistulas. At this time, the main idea is to reduce the size of the neoplasm and heal the wound, and then removing the lesions of the breast tissue should be followed. For the reduction of size of tumors, the mainstream method of Western medicine is hormone therapy [27]. In a metaanalysis [8], 106 patients were analyzed for surgical managements, oral steroids, and oral steroids + surgical managements, respectively. The pooled estimates for their CR rate were $90.6 \%, 71.8 \%$, and $94.5 \%$. The pooled estimates for recurrence rate were $6.8 \%, 20.9 \%$, and $4.0 \%$, respectively. The present study uncovered that IGM had a tendency of self-healing, which was consistent with Lei et al.'s findings [8]. However, we also found that the severity of pain should be take into consideration in treatment of IGM. Our results indicated that CLY had a significant effect on pain relief in IGM patients.

The use of herbal TCM to treat various diseases has an interesting philosophical background with a long history, while it received increasing skepticism due to the lack of evidence-based efficiency as shown by high quality trials. Our previous study revealed that CLY had an acceptable anti-inflammatory effect, promoting blood circulation and wound healing [13]. In the present study, we found that CLY could inhibit the levels of inflammatory factors, such as TNF- $\alpha$, IL- $1 \beta$, and IFN- $\gamma$, suggesting that CLY could promote wound healing by inhibiting the levels of inflammatory factors.

IL-2 is a pleiotropic cytokine produced after antigen activation that plays pivotal roles in the immune response [28]. IL-2 can promote the generation of $\mathrm{T}$ effector cells including those of the Th1 and Th2 lineages, while inhibiting the differentiation of Th17 cells [29]. Although there is no literature supporting the role of IL-2 in granulomatous mastitis, our experiment found that there was no significant difference between the two groups before treatment. After treatment, the $P$ value of IL- 2 was 0.057 , and there were still some differences although that was greater than 0.05 . This indicated that CLY may play a substantial role in the immune response to IGM, while further study needs to be conducted to confirm our findings.

There are a number of limitations in the current study. First, lack of long-term follow-up of recurrence rate was tangible. Secondly, IL-6 is also an important cytokine, and it was previously reported that IL-6 plays a key role in the development of IGM $[13,30]$. Thirdly, our findings unveiled that CLY had insignificant influence on IL-6 level, which is worthy of further discussion.

In summary, our findings proved that CLY could promote the healing of the neoplasm and alleviate the pain in patients with IGM.

\section{Abbreviations}

CLY: $\quad$ Chuang Ling Ye

IL-2: Interleukin-2

IL-6: Interleukin-6

IL-1 $\beta$ : Interleukin- $1 \beta$

IFN- $\gamma$ : Interferon gamma

TNF- $\alpha$ : Tumor necrosis factor- $\alpha$

IGM: Idiopathic granulomatous mastitis.

\section{Data Availability}

The data used to support the findings of this study are available from the corresponding author upon request.

\section{Conflicts of Interest}

The authors declare that there are no conflicts of interest regarding the publication of this manuscript.

\section{Authors' Contributions}

Jing-xian Xue and Bei Ye contributed equally to this work. $\mathrm{XJX}, \mathrm{YB}$, and BWH designed the study. XJX, LS, and CSH 
recruited and screened the patients, and performed bioassays. CSH and LS carried out the biochemical analysis. YB and $\mathrm{BWH}$ performed statistical analysis. XJX and BWH wrote the manuscript. All the authors read and approved the final version of the manuscript.

\section{Acknowledgments}

This study was financially supported by the Natural Science Foundation of Jiangsu Province, China (grant no. BK20191058), and Advanced Constructed Discipline of Universities in Jiangsu Province (grant no. ZYX03KF024).

\section{References}

[1] R. A. Patel, P. Strickland, I. R. Sankara, G. Pinkston, W. Many, and M. Rodriguez, "Idiopathic granulomatous mastitis: case reports and review of literature," Journal of General Internal Medicine, vol. 25, no. 3, pp. 270-273, 2010.

[2] J. Li, "Diagnosis and treatment of 75 patients with idiopathic lobular granulomatous mastitis," Journal of Investigative Surgery, vol. 32, no. 5, pp. 414-420, 2019.

[3] R. Omranipour, S.-F. Mohammadi, and P. Samimi, "Idiopathic granulomatous lobular mastitis-report of 43 cases from Iran; introducing a preliminary clinical practice guideline," Breast Care, vol. 8, no. 6, pp. 439-443, 2013.

[4] S. Akbulut, D. Yilmaz, and S. Bakir, "Methotrexate in the management of idiopathic granulomatous mastitis: review of 108 published cases and report of four cases," The Breast Journal, vol. 17, no. 6, pp. 661-668, 2011.

[5] J. R. Benson and D. Dumitru, "Idiopathic granulomatous mastitis: presentation, investigation and management," Future Oncology, vol. 12, no. 11, pp. 1381-1394, 2016.

[6] P. Chirappapha, P. Thaweepworadej, C. Supsamutchai, N. Biadul, and P. Lertsithichai, "Idiopathic granulomatous mastitis: a retrospective cohort study between 44 patients with different treatment modalities," Annals of Medicine \& Surgery, vol. 36, pp. 162-167, 2016.

[7] F. N. L. Versluijs-Ossewaarde, R. M. H. Roumen, and R. J. A. Goris, "Subareolar breast abscesses: characteristics and results of surgical treatment," The Breast Journal, vol. 11, no. 3, pp. 179-182, 2005.

[8] X. Lei, K. Chen, L. Zhu, E. Song, F. Su, and S. Li, “Treatments for idiopathic granulomatous mastitis: systematic review and meta-analysis," Breastfeeding Medicine, vol. 12, no. 7, pp. 415-421, 2017.

[9] C. Yao, L. L. Chen, Y. P. Li, C. Z. Peng, M. K. Li, and J. Yao, "Multi-variated analysis of differential diagnosis in ultrasonography of idiopathic granulomatous mastitis and invasive ductal carcinoma," Zhonghua Zhong Liu Za Zhi, vol. 40, no. 3, pp. 222-226, 2018.

[10] D. Lv, X. Chen, N. Zhang, X. Wu, and M. Jiang, "Total flavone of Abelmoschl manihot L. medic exhibits protective effect against hind-limb ischemia in rat model," Pakistan Journal of Pharmaceutical Sciences, vol. 32, no. 1, 5 pages, 2019.

[11] L. Tang, W. Pan, G. Zhu, Z. Liu, D. Lv, and M. Jiang, "Total flavones of abelmoschus manihot enhances angiogenic ability both in vitro and in vivo," Oncotarget, vol. 8, no. 41, pp. 69768-69778, 2017.

[12] J. B. Song, Z. H. Wang, J. Q. Ren et al., "Development and clinical application of Chuang ling Ye," Shi Zhen Guo Yi Guo Yao, vol. 7, pp. 5-6, 1996.
[13] L.-L. Wu, X.-B. Yang, Z.-M. Huang, H.-Z. Liu, and G.-X. Wu, "In vivo and in vitro antiviral activity of hyperoside extracted from Abelmoschus manihot (L) medik," Acta Pharmacologica Sinica, vol. 28, no. 3, pp. 404-409, 2007.

[14] D. Zhang, P. Zhu, Y. Liu et al., "Total flavone of Abelmoschus manihot ameliorates Crohn's disease by regulating the NFkappaB and MAPK signaling pathways," International Journal of Molecular Medicine, vol. 44, pp. 324-334, 2019.

[15] Y. Tu, W. Sun, Y.-G. Wan et al., "huangkui capsule, an extract from abelmoschus manihot (L.) medic, ameliorates adriamycin-induced renal inflammation and glomerular injury via inhibiting p38MAPK signaling pathway activity in rats," Journal of Ethnopharmacology, vol. 147, no. 2, pp. 311-320, 2013.

[16] Chinese Pharmacopoeia Commission, Pharmacopoeia of the People's Republic of China, vol. 1, pp. 292-293, China Medical Science Press, Beijing, China, 2015.

[17] D. Anton, J. Koskar, P. Raudsepp et al., "Antimicrobial and antioxidative effects of plant powders in raw and cooked minced pork," Foods, vol. 8, no. 12, p. 661, 2019.

[18] T.-Z. Yang, Y. Liu, Y.-Y. Liu et al., "The use of Rheum Palmatum L. in the treatment of acute respiratory distress syndrome: a meta-analysis of randomized, controlled trials," African Journal of Traditional, Complementary and Alternative medicines, vol. 14, no. 2, pp. 334-347, 2017.

[19] Y. A. Jahani, S. Javanshir, M. Soukhtanloo et al., "Acute and sub-acute toxicity evaluation of the root extract of Rheum turkestanicum Janisch," Drug and Chemical Toxicology, pp. 1-7, 2019.

[20] Z.-J. Pu, S.-J. Yue, G.-S. Zhou et al., "The comprehensive evaluation of safflowers in different producing areas by combined analysis of color, chemical compounds, and biological activity," Molecules, vol. 24, no. 18, p. 3381, 2019.

[21] E. C. de Ataide, S. Reges Perales, M. A. de Oliveira Peres et al., "Acute liver failure induced by Carthamus tinctorius oil: case reports and literature review," Transplantation Proceedings, vol. 50, no. 2, pp. 476-477, 2018.

[22] H. K. Chen, D. Lee, H. P. Kim, and S.-H. Baek, "Structure analysis and antioxidant activities of an amylopectin-type polysaccharide isolated from dried fruits of terminalia chebula," Carbohydrate Polymers, vol. 211, pp. 100-108, 2019.

[23] A. Bag, S. K. Bhattacharyya, and R. R. Chattopadhyay, "The development of Terminalia chebula retz. (combretaceae) in clinical research," Asian Pacific Journal of Tropical Biomedicine, vol. 3, no. 3, pp. 244-252, 2013.

[24] N. Sanchez-Zauco, J. Torres, A. Gomez et al., "Circulating blood levels of IL-6, IFN-gamma, and IL-10 as potential diagnostic biomarkers in gastric cancer: a controlled study," BMC Cancer, vol. 17, no. 1, p. 384, 2017.

[25] A. Wolfrum, S. Kümmel, I. Theuerkauf, E. Pelz, and M. Reinisch, "Granulomatous mastitis: a therapeutic and diagnostic challenge," Breast Care, vol. 13, no. 6, pp. 413-418, 2018.

[26] T. D. Lehman, Z. Damania, C. N. Tschetter, and N. L. Lehman, "Firm, non-tender neoplasm in right breast worsening, nonproductive cough pleuritic pain Dx?" The Journal of Family Practice, vol. 66, pp. 38-41, 2017.

[27] M. Co, V. C. C. Cheng, J. Wei et al., "Idiopathic granulomatous mastitis: a 10-year study from a multicentre clinical database," Pathology, vol. 50, no. 7, pp. 742-747, 2018.

[28] J. Clark, P. Vagenas, M. Panesar, and A. P. Cope, "What does tumour necrosis factor excess do to the immune system long term?" Annals of the Rheumatic Diseases, vol. 64, no. 4, pp. iv70-iv76, 2005. 
[29] O. Boyman and J. Sprent, "The role of interleukin-2 during homeostasis and activation of the immune system," Nature Reviews Immunology, vol. 12, no. 3, pp. 180-190, 2012.

[30] Y. Liu, J. Zhang, Y.-h. Zhou et al., "Activation of the IL-6/ JAK2/STAT3 pathway induces plasma cell mastitis in mice," Cytokine, vol. 110, pp. 150-158, 2018. 